\title{
Smart Phone Sensing to Examine Effects of Social Interactions and Non-Sedentary Work Time on Mood Changes
}

\author{
Alelsandar Matic, Venet Osmani, Andrei Popleteev, Oscar Mayora-Ibarra \\ CREATE-NET,Via alla Cascata 56d/ 38123 Povo, Trento, Italy \\ name.surname@create-net.org
}

\begin{abstract}
Study of mood and in turn mood changes is an important index of general wellbeing state and can also be an indicator of various mood disorders including clinical depression and bipolar disorder. While there have been clinical studies of mood, less emphasis has been placed on the factors that affect the mood, especially in workplace. The typical approach taken by these studies is to use clinical questionnaires in order to record the activities that impact the mood. However, recording activities that may have an influence on the mood in this manner suffers from a number of issues including floor effect and difficulty in recalling past activities. Our work instead has focused on using unobtrusive monitoring technology to study mood changes during office hours and a set of associated factors that influence these changes. We have primarily studied two factors and their impact on the mood changes, namely social activity and nonsedentary work style. The pilot study ran over the course of 7 days of measurements with the participation of 9 knowledge workers. The results have shown that mood changes are highly correlated with both social interactions and non-sedentary work style. This study is the first to investigate the correlation between mood changes and non-sedentary behavior patterns, opening up a research avenue to explore psychological effects of increasing prevalence of sedentary behavior.
\end{abstract}

Keywords: Sedentary behavior, mood changes, accelerometers, sitting, social interactions, activity recognition, pervasive computing.

\section{Introduction}

As computational capabilities continuously improve and sensing devices shrink in size, the use of technology is enabling monitoring many aspects of individuals' life, from day to day activities up to various physiological parameters. This allows a number of applications that improve the quality of life of people by assisting them in every-day life activities or sending an alarm in the case of emergency. Furthermore the technology provides more objective and precise insight into domains that are difficult or even impossible to be monitored by a human observer. This affords acquiring new theoretical knowledge in various sciences such as medicine, sociology, psychology and psychiatry. A number of examples of using technology to measure new socio-medical phenomena can be found in the literature including Hasler et al. 
[26] that used automatic sound analysis to detect sinusoidal $24 \mathrm{~h}$ rhythms in activities associated with positive affects. Using data from various sensors, Olguin et al. [39] measured and modeled organizational behavior at the individual and group levels. Fishbah et al. [40] employed the social badge, developed by MIT lab, to analyse informal social networks in companies aiming to increase employees' productivity. RFID-based indoor localization system enabled Kearns et al. [41] to correlate the moving patterns and the degree of cognitive impairment which is a foundation for the preventative system that can detect early stages of dementia.

Despite the research work and advancements in technology, clinical studies still rely mostly on self-reporting methods to describe past activities. These methods have a number of drawbacks, including floor effect and difficulties in recalling activities that have occurred in the past [42]. In particular, studies that capture mood changes and the associated factors typically suffer from subjectivity of perception regarding the factors that affected the mood and difficulty in recalling these factors. Mood changes during work time and the associated factors that affect the mood have not been studied in detail using advantages brought by context-aware applications.

In the current study, we attempt to automatically recognize behavioral parameters and investigate their influence on the mood of knowledge workers. Clearly, the potential number of factors that can affect the mood is high and some of these factors are difficult to be monitored directly. However, in our study we focused on external behaviour that can be directly measured using smart phone, namely sedentary patterns and social interactions, and how such behaviour affects the mood. In particular, for the purpose of this work we recognized a set of pleasant social interactions, namely interactions that occurred during coffee and snack breaks. The term coffee/snack break refers to the place where the interactions occurred during work time; therefore, for the rest of the paper we will refer to these as breaks. The survey, which will be presented later in the paper, shows that these short breaks are perceived as pleasant social interactions by participants in our experiments. In addition to social interactions the study was then extended to include sedentary patterns for two reasons: 1) sedentary behavior has been linked with insulin sensitivity [10], lipid metabolism [10] and sympathetic activity [44], which influence the mood, as shown through a number of clinical studies [44, 45, 46]; thus it was seen as a potential factor that may also affect the mood; 2) related to socialization patterns is also the behavior of workers' during workday, specifically number of overall breaks and their duration.

Study of mood changes and factors that influence these changes can provide a good estimation of the general wellbeing of the knowledge workers and avoid lost productive time. Mood changes affect work productivity and according to a study in [7], US workers with depressed mood reported much more Lost Productive Time (LPT) than those without depression (mean 5.6 hours/week versus 1.5 hours/week respectively), which costs employers an estimated $\$ 44$ billion per year in LPT. More critically however, mood changes are associated with various disorders including bipolar disorder, clinical depression and mania.

The aim of this study was twofold: $i$ ) investigate correlation between measured social activity and self-reported mood changes; and ii) stemming from social activity, study of sedentary patterns and their influence on mood. Together, the results have shown an evident correlation between the reported mood changes and socialization activities and also between non-sedentary work style and mood changes. This study provides a 
basis to establish new health recommendations and create work place policies that minimize sedentary work style, so that the wellbeing of knowledge workers is further improved. Use of mobile phones will allow workers to get feedback on their mood changes scores and correlation with non-sedentary periods thus forming a part of persuasive feedback application that may be developed to encourage a healthier work style.

Before delving into the description of the results, we first review the current state of the art in this area.

\section{Related work}

This section reviews the current literature related to our work concerning mood variation studies, technological solutions applied for inferring social behaviour and research in sedentary lifestyle.

Robbins et al. [3] explored patterns of depressed mood periods in normal subjects with the goal of following diurnal variations as a function of time of the day. The study demonstrated mood swings in eighty-four percent of the days in which the subjects reported depressed state. Individuals who reported days of constant depressed mood emphasized higher levels of physical symptoms and less pleasure in social interactions. On the other hand, Volkers et al [9] argued that it is not possible to clearly infer how mood states are related to time of the day since conflicting findings were reported about the timing of mood highs and lows and the pattern of variations during the day. In addition, the authors identified the sleep quality as one of the factors related to disturbances of mood. Subjective sleep quality was assessed every morning by a brief self-rating questionnaire while the adjusted version of the profile of mood states served for the evaluation of the mood after awakening and during morning, afternoon and evening. They claim that additional analyses are needed to take into account the influences of work and leisure time activities. Stone et al [30] focused on activities and locations as determinants of daily mood variability. They discovered diurnal cycles for several moods, activities and locations and managed to correlate most moods with certain activities and locations. Vittengl and Holt [1] investigated correlations between social interactions and NA/PA (Negative affect/Positive affect). Both mood and assessment of social interactions were selfreported using structured diaries. Positive correlations were found between PA and fun/active and necessary/informational types of social interaction and between NA and arguing/confronting and receiving help/support. No evidence was discovered between providing help/support and NA or PA.

The above-mentioned studies applied methods of self-reporting for examining both psychological parameters and behavioral/environmental factors in order to uncover relations between the two. Concerning mood related research that exploits technological solutions, the goal was mostly to understand human affective behaviour and to recognize emotions, usually relying on video and audio sensors. Related work in this field is comprehensively covered in the survey carried out in [27]. However, yet not many studies have used technology to investigate determinants of psychological responses. One of those studies is elaborated by Hasler et al. [26] who 
examined daily variability in behaviour associated with PA and NA using Electronically Activated Recorder (EAR). By using that device to record ambient sounds, authors found that activities associated with PA (e.g. socializing, laughing and singing) varied according to a sinusoidal $24 \mathrm{~h}$ rhythm centered around participants' average wake time, while that was not the case for activities usually associated with NA (e.g. arguing and sighing). In regards to investigating sociability at workplace, the study in [40] focuses on productivity and its relation to informal social networks at workplace. The approach was based on using the Social Badge developed by MIT Media Lab, capable of monitoring the geographic position (Bluetooth), direction of movement and speed (tri-axial accelerometer), interaction (infrared sensor) and mood (microphone and speech analysis). The authors used the Social Badge in combination with social network analysis methods in order to determine subject's communication behaviour and to reconstruct the social network.

A number of studies investigated sedentary behaviour and its implications on health. In the recent survey of the literature carried out in [10], authors reviewed work related to implications of sedentary lifestyle on health problems, specifically metabolism, bone mineral content, obesity and vascular diseases. However, the main focus was mainly on physiological consequences while no study that addresses correlations between sedentary behaviour and mood is reported. Hence, we do not provide here more extensive state of the art on that topic.

There are no studies reported in the current literature that employ technological solutions to infer correlations between mood changes and socialization patterns. Furthermore, this work is the first to investigate the influence of sedentary behaviour on mood changes, which may provide the motivation for further investigation of psychological implications of increasing prevalence of sedentary work style.

\section{Study of mood changes and associated factors}

One's mood may depend on a number of different factors, such as circadian rhythms [13], type of environment [14], quality of sleep [9], state of health, private problems or some other factors incomprehensible not only through direct measurement but also difficult for an individual himself/herself to identify. Therefore, it may be impossible to consider all the factors that influence the mood and provide the ultimate conclusion about the exact cause of one's state of mood. For this reason, our approach is to follow relative changes of mood rather than focus on an absolute mood state, where we assume that interval between two mood assessments of a couple of hours (in our design) is not sufficient for a significant change in "background" factors. These factors, such as private problems for example, are likely to be constantly present during relatively longer periods of time while, the activities within that period have pre-dominant influence on relative changes of mood. The goal of our research is to capture patterns of these activities that, in most cases, provoke similar responses in individuals' mood.

In our study we chose to investigate how non-sedentary behavior and social contacts, perceived by the workers as pleasant, impact their mood. Typically, the study of mood changes is carried out through questionnaires in a clinical setting in which 
participants describe past activities. However, we have attempted to make this approach as unobtrusive as possible to minimize the impact on workers' routines, which may affect their mood and thus potentially decrease the chances of gathering representative results.

People cannot categorize past actions and their duration in an accurate manner as monitoring technology can (for instance, the exact duration of breaks or their mobility patterns). Moreover, people are prone to neglect certain parameters that influence their mood or overestimate the influence of other aspects (such as a very small effect of weather in individuals' day-to-day mood despite the commonly held conception that weather greatly affects the mood [8]). Large majority of the studies that addressed determinants of daily variations in psychological responses relied on selfadministered questionnaires to record past activities, which presented difficulties in this research field. For example Clark and Watson [5, 6] argue that no such relation exists between NA and social activity, which is an opposing view to other studies [1, 3, 4], while Volkers at al. [9] claim that is not possible to clearly infer how mood states are related to the time of the day which was concluded by a number of other reports. In the paragraphs that follow we describe the two factors that were monitored during our study of mood changes, namely social activity and non-sedentary work style.

\subsection{Social Activity}

Empirical research has suggested that forms of social interactions and different mood states are interrelated [1]. It is widely accepted that social interaction and the way one feels are related [1]. Happy persons are more likely to involve themselves in social interactions than those who are feeling sad [2], and it is commonly held that talking to a friend about personal problems can make one feel better. In addition, several clinical studies (refer to Section 2) have found that social contacts exert influence on the state of mood in people.

Social interaction is typically an integral aspect of work, involving different types of conversations - from informal chats between colleagues to official meetings, negotiations or interviews. Therefore, social interaction can be perceived from workers' point of view both as a pleasant experience but also as a displeasing one (for example, an informal chat with colleagues versus an imposed meeting or an agreement from co-workers versus having an argument). This makes the workplace a source of stimulus both for positive and negative emotions.

In order to recognize a set of pleasant social interactions and investigate how they affect workers' mood, we based our approach on the location recognition, in particular focusing on break room and balconies. These are the locations where workers typically have breaks during working time and have the opportunity for a relaxed conversation with colleagues which was confirmed by the results of the survey (presented in Section 4.6). Our monitoring framework recognizes with a high certainty the location of the subjects, when they are in the break room, meeting room or on the balconies; therefore, as opposed to self-reporting methods, the monitoring system provides much more reliable and precise information about workers' behavior while not intruding in their routines. In this manner, it is possible to isolate, with a 
high probability, a part of pleasant social interactions and to assess their influence on the mood.

In addition to examining the effect of social interactions on workers' mood, we also analyse the duration of non-sedentary time in general and study the ratio between the time spent on breaks and sedentary periods during working time. According to our findings, sedentary patterns impact the mood and the section that follows describes this study.

\subsection{Sedentary work style}

Sedentary behavior (from the Latin "sedere" meaning "sitting") refers to the category of behaviors for which the energy expenditure is low, including prolonged sitting periods at work, home, in car, bus, and in leisure time [10]. Sedentary behavior, characterized by the SITT formula (Sedentary behavior frequency, Intensity, Time and Type), is often associated with deleterious health outcomes. These health outcomes are of different nature from causes attributed to a lack of moderate or intense physical activity outdoors [10]. Therefore, deleterious health outcomes still occur as a result of sedentary behavior, even if guidelines for physical activity are respected. Emerging studies suggest that independently and qualitatively different from a lack of moderate or intense physical activity, sedentary behavior has effects on human metabolism, physical function and health outcomes and therefore it should be considered as a separate and unique phenomenon [10]. Even if one meets physical activity guidelines, sitting for prolonged periods increases the risks for obesity, metabolic syndrome, type 2 diabetes and cardiovascular disease [10, 47].

Previous research initiatives have relied on self-reporting methods to acquire information about activities that correspond to sitting routines. On the other hand, accelerometers can provide recognition of physical activities such as sitting, running, standing $[11,12]$ and in addition record further details such as duration, frequency and intensity of movements. Accelerometers have become critical in investigation of sedentary habits that are difficult to recall with the questionnaire method. With the advancement of technology, accelerometers have been used to capture sedentary patterns in an objective manner, thus overcoming the drawbacks of self-reporting. Usage of accelerometers allowed significant results in establishing the influence of sedentary behavior on metabolism [15], vascular health [15] and bone mineral content [16]. Moreover, recent studies link sedentary lifestyle and obesity [17], cancer [18] and psychosocial health [19]. Sanchez-Villegas et al. [20] found that the risk of mental disorders was $31 \%$ higher for prolonged patterns of sitting in front of a computer or a TV, comparing subjects that spend more than $42 \mathrm{~h} /$ week in front of a TV with those watching less than $10.5 \mathrm{~h} /$ week. The extensive literature survey on the implications of sedentary lifestyle, provided by Tremblay et al. [10], concludes that there is a need to understand the factors of sedentary behavior and to implement interventions to reduce population-wide levels of sedentary behavior, shifting some proportion of time from sitting to various types of physical activity. The authors [10] suggest that this should be done empirically.

By conducting an empirical study that investigates non-sitting periods during working time, we have studied correlation between non-sedentary time at workplace and office 
workers' mood changes, which can form the basis for workplace policies to reduce prolonged sitting periods. This work is the first to investigate the influence of nonsedentary behavior on mood changes, opening up a research avenue to explore psychological effects of increasing prevalence of sedentary habits.

\section{Monitoring Setup}

The goal of the reported work was to design a monitoring framework that can monitor when subjects have breaks and infer their sedentary patterns during working time. Our conceptual idea was to monitor primarily two behavioral aspects, namely subjects' location (such as break room, balcony, office or meeting room) and the amount of physical activity. Clearly, the monitoring technology should be as invisible as possible, while not imposing additional sensing devices that subjects would need to carry. Therefore, mobile phone, as widely accepted and commonly used by office workers, was an appropriate solution. Although the technology of smart sensing is moving towards further miniaturization, bringing a variety of embedded sensors, the challenge was to allow monitoring aforementioned parameters solely using a mobile phone. In the following sub-sections we detail our approach.

\subsection{Non-Sedentary time}

Accelerometers provide an important research tool able to reliably measure and classify a number of physical activities, including walking, jogging, sitting, standing [12], and more complex activities such as estimation of metabolic energy expenditure, sit-to stand transfers, and assessment of balance and intensity of physical activity [11]. In our study we focused on distinguishing between sedentary and non-sedentary time. Typical approach is recording accelerometer data in 1-min epochs and a threshold of $<100$ counts per minute (CPM) is chosen to classify sedentary time [10, 48]. Total sedentary time is calculated as a sum of all sedentary minutes, while each minute interval where number of accelerometer counts is above 100 is considered a non-sedentary break [48]. The current studies investigating sedentary behavior typically use dedicated devices such as ActiGraph, TriTrac, Caltrac, Actiwatch or Actical [48] that directly provide the number of counts.

In our study we opted for a mobile phone, not only because it does not require an additional sensing device to be carried, but also it does not impact the typical behavior routines of knowledge workers. In addition accelerometers are widely available in smart phones, due to their role in user interfaces [25]. Estimating physical activity using smart phones is usually based on the analysis of the raw accelerometer signal. In order to characterize activities, various features extracted from the signal are considered, including average acceleration, standard deviation, average absolute difference, average resultant acceleration, time between peaks and binned distribution [12]. In our study, distinguishing sedentary and non-sedentary time a simpler approach sufficed - we considered standard deviation (SD) of resultant accelerations over each one-minute interval - the square roots of the sum of the values of each axis 
( $x, y$ and $z$ ) squared [11]. However, we compared the performance of the two approaches for inference of sedentary time in our experimental settings, namely SD analysis and CPM-based method.

Each time the measured acceleration value deviates from the normal gravitational acceleration $g$ by more than a certain threshold it is considered a count [49]. Consistent with previous research, we summed counts in 1-min epochs and applied the widely accepted criteria for classifying sedentary time ( $<100$ counts per minute). However, even off-the-shelf accelerometers differ in reported counts when evaluated together for the same activities over the same period, which may result in under- or overestimation of monitored activities [49]. Therefore, we found it necessary to calibrate the algorithm to calculate the counts in order to account for specific parameters of the used accelerometer. We performed a separate experiment with different motions and activity levels, recording the accelerometer data and manually labeled the ground truth. From our experiment, the acceleration threshold of $0.75 \mathrm{~g}$ was found to provide the optimal sensitivity of counts to activity levels.

Within the same experiment, we compared the accuracy of SD analysis and counts per minute $(\mathrm{CPM})$ to infer sedentary time and found the former to be more sensitive to low intensity motion.

Table 1. Accuracy of the approach based on SD Analysis.

\begin{tabular}{|l|l|l|}
\hline Activity & Sedentary Activity & Non Sedentary Activity \\
\hline Sitting & $92.5 \%$ & $7.5 \%$ \\
\hline Walking & $0 \%$ & $100 \%$ \\
\hline Walking/ Standing & $17 \%$ & $83 \%$ \\
\hline
\end{tabular}

Table 2. Accuracy of the approach based on CPM

\begin{tabular}{|l|l|l|}
\hline Activity & Sedentary Activity & Non Sedentary Activity \\
\hline Sitting & $100 \%$ & $0 \%$ \\
\hline Walking & $0 \%$ & $100 \%$ \\
\hline Walking/ Standing & $29 \%$ & $71 \%$ \\
\hline
\end{tabular}

Considering the knowledge workers' routines the analyzed activities included: a) sitting (considering the phone placed on the desk and in the subject's pocket/case), b) walking (with different speed), c) alternately walking and d) standing. Table 1 and Table 2 show confusion matrixes for both approaches. Being more sensitive to low intensity movements while subject is standing, SD approach correctly classified walking/standing as non-sedentary in higher number of cases than CPM method. The approach based on CPM was more accurate in inferring sedentary time due to its robustness on low intensity movements, typical for events when using the phone which resulted in false negatives for SD analysis. However, activities related to the phone use such as making phone calls, sending SMS or filling out the questionnaires 
were reported by a monitoring application during the experiments and the matching timestamps, when these activities occurred, were used to discard the associated accelerometer data. In this way, it was possible to correct false negatives in sitting activity detection in the case of SD analysis, which resulted in better overall performance of this method in comparison to CPM. Therefore, the recognition of sedentary/non-sedentary time was based on standard deviation analysis. Clearly, an issue with technological monitoring is that subjects may not always wear the device and this is an issue that we will address in the upcoming studies. However, the accelerometer logs have not shown periods of total inactivity, indicating that subjects kept the phone with themselves (such as in the pocket). The typical approach to deal with this issue, by using a 20-minute inactivity criterion to identify a non-wearing period was not applicable in our study as office workers may sit continuously for more than 20 minutes.

\subsection{Location}

We defined the following set of locations that were deemed important to monitor: break room, offices, meeting room, balconies and outside of the workplace. Within the scope of our study we aimed to recognize the duration of time in which subjects are in the break room or on the balconies. Since GPS is unusable indoors, another localization system that was accurate enough was needed, with the constraint of being available using solely a mobile phone. As an effective solution, we used our FM radio-based positioning system [21] that provides the median accuracy of around $1 \mathrm{~m}$, and it is convenient for installation in various environments. The mobile phone used in the tests was a Samsung i8000 with Windows Mobile 6.5 operating system, with MyExperience tool installed and with an embedded accelerometer and an FM receiver. Nonetheless, the positioning system is easily applicable for many smart phones with built-in FM radio receiver.

\subsection{Measuring mood changes}

Our method for assessing mood fluctuations during the day is based on EMA (Ecological Momentary Assessment) approach in order to compare retrospective and momentary mood data [50]. The EMA approach, which involves asking participants to report their psychological state multiple times a day, reduces the critical issue of retrospective recall of extended time intervals. The retrospective recall issue is related to cognitive and emotive limitations that bias the recall of autobiographical memory [50] influencing subject's report by most salient events during the recall interval. The questionnaire we used was derived from a well-established scale for mood study - the Profile of Mood States (POMS) scale that consists of 65 items in its standard version. However, long and repeated mood questionnaires become a burden on the subjects, therefore a short version of the POMS scale was used. In a similar way to the study conducted by Steptoe and Wardle [23], we derived 8 adjectives from the POMS scale (cheerful, sad, tensed, fatigued, energetic, relaxed, annoyed, friendly) used a 5-point scale and averaged the items related to positive and negative expression in order to 
generate a single scores. The difference of these scores (between two sequential questionnaires) was taken as measures of relative change of subject's mood. The questionnaires were administered three times a day, scheduled to best fit with the work routines, while also allowing the user to manually invoke the questionnaire. Typically, the questionnaires were answered in the morning, after lunch and at the end of working day.

\subsection{Subjects}

We recruited 9 participants from our research center (6 males, 3 females), not connected with this study, for 7 working days within a period of one month (characteristics of the sample is shown in Table 3). The participants filled out the mood questionnaires in the beginning, in the middle and at the end of working day. There were no significant differences between men and women either in the relevant parameters (such as age, number of working hours or type of the job regarding sedentary routines) or in the measures (such as a number of reported positive/negative mood changes, average non sedentary time within one monitored interval or average number of breaks). None of the participants was a cigarette smoker nor reported health problems.

Table 3 Characteristics of the sample

\begin{tabular}{ll}
\hline Age (years) & $28.4 \pm 2.8$ \\
Marital status & \\
Married & $11 \%$ \\
$\quad$ Single, Divorced & $89 \%$ \\
University/post diploma & $77 \%$ \\
Work hours/week & $36.6 \pm 4.6$ \\
Duration between two questionnaires (minutes) & $174.3 \pm 49.8$ \\
$\quad$ Morning intervals (minutes) & $187.4 \pm 47.5$ \\
$\quad$ Afternoon intervals (minutes) & $161.3 \pm 48.5$ \\
Non-sedentary time in one interval (minutes) & $35.4 \pm 18.2$ \\
Number of breaks in one interval & $1.6 \pm 0.7$ \\
Number of reported positive mood changes & $5.3 \pm 1.7$ \\
Number of reported negative mood changes & $5.7 \pm 2.1$ \\
\hline
\end{tabular}




\subsection{Data and Analysis}

After discarding intervals due to non-completed reports, the data analyzed contained 112 monitored intervals, 54 and 58 intervals of positive and negative mood changes respectively. The overall duration of the recorded data was 339.8 hours, $181.2 \mathrm{~h}$ in morning intervals (between first and second questionnaire) and 158.6h in afternoon intervals (between second and third questionnaire). The mean score for positive, negative and overall mood score was $2.89 \pm 0.65,2.12 \pm 0.67$ and $0.76 \pm 1.19$ respectively. The mean within-subject correlation between positive and negative, positive and overall, negative and overall mood score was $0.147 \pm 0,094,0.734 \pm 0.102$ and $0.511+0.211$ respectively. Self-reported mood change, measured as a difference in scores between two consecutive questionnaires, was analyzed with respect to nonsedentary time and a number of breaks. Spearman correlations were calculated between non-sedentary time and mood scores and between a number of breaks and mood scores on a within-subject basis. Statistical analysis was performed using SPSS (SPSS, Inc. Chicago) while sedentary patterns and locations were inferred with R2.13 .

\subsection{Background Measures}

In order to investigate our assumption that social interactions during breaks are perceived in a positive way, we conducted a survey among 15 colleagues and among participants in the study. We asked them to rate on a 5-point scale (from "not at all" to "very") the statement: "Social interaction during breaks is mostly pleasant for me". The mean score was 3.87 for a randomly chosen sample and 4.00 for participants of our study, varying in answers only from 3 to 5 . Furthermore, both groups were asked to indicate the approximate percent of cases when they are going to a break with someone rather than alone. The possible answers were "<50\%", "50-60\%", "60$70 \% "$ " "70-80\%", "80-90\%" and "90-100\%" and the results of this survey are presented on Fig. 1. 


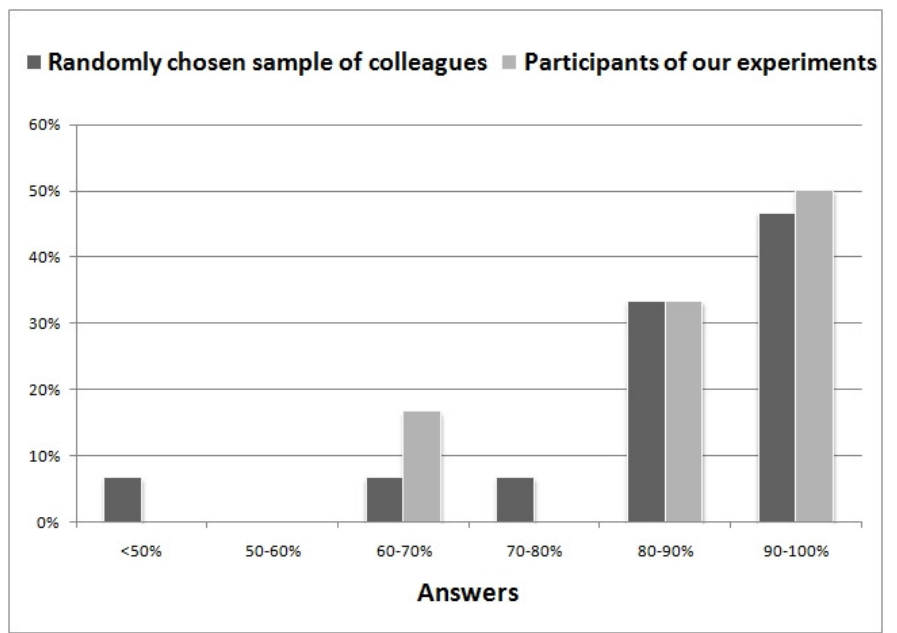

Figure 1 Proportion of participants vs. Frequency of accompanied breaks

These results show that breaks are centered on social interactions, considering that $50 \%$ of the participants surveyed are very likely (90-100\%) to go on a break with someone, while another $30 \%$ go with someone in $80-90 \%$ of cases. Only one participant reported that he goes on a break alone.

\section{Findings}

\subsection{Correlations between non-sedentary time and mood changes}

Figure 2 shows the distributions of Spearman correlation between non-sedentary time and reported change in mood. The mean correlation between non-sedentary time and positive/negative/overall mood changes was $0.24 \pm 0.26$ ( $\min =-0.01, \max =0.81$ ), $-0.26 \pm 0.36 \quad(\min =-0.91, \quad \max =0.33)$ and $0.38 \pm 0.32 \quad(\min =-0.02, \quad \max =0.86)$ respectively. 

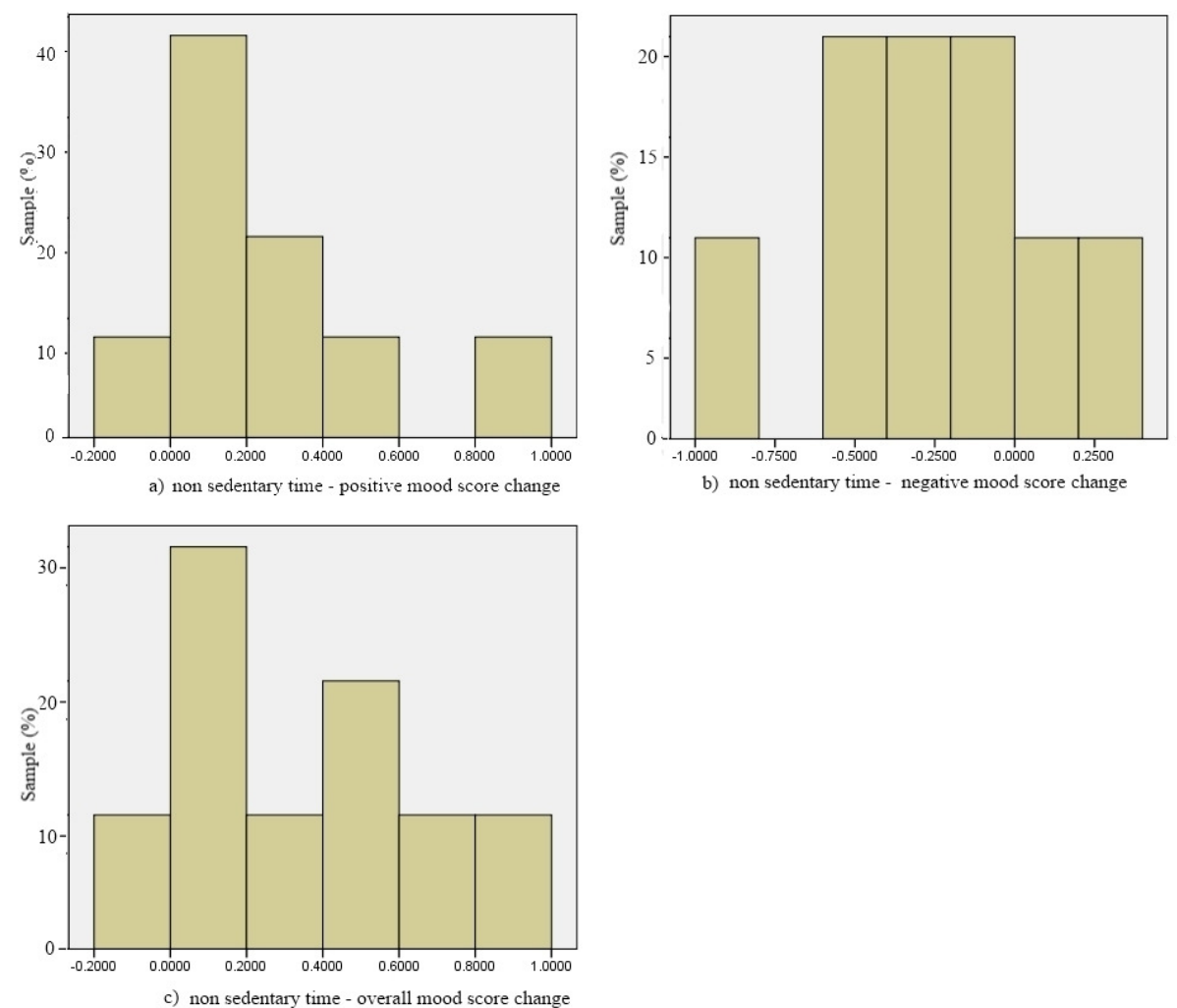

Figure 2 Distributions of Spearman correlations between non-sedentary time and a) positive mood, b) negative mood and c) overall mood score change

The non-sedentary time/positive mood changes and non-sedentary-time/overall mood changes distributions were significantly greater than $0(t=2.925, t=3.588$ respectively, $\mathrm{P}<0.01$ ) while non-sedentary time/negative mood changes distribution was significantly less than $0(t=-2.392 \mathrm{P}<0.025)$. None of the distributions was significantly skewed. The results suggest that the time spent in non-sedentary activities is positively correlated with changes in reported positive/overall mood and negatively correlated with changes in reported negative mood. On the other hand, the reported mood at the beginning of monitored intervals had moderately low impact on the non-sedentary behavior in the following interval $(r=0.27, r=-0.18$ and $r=0.24$ respectively for initial positive/negative/ overall mood score and non-sedentary time across subjects). This suggests that sedentary patterns were not influenced by subjects' mood.

\subsection{Correlations between breaks and mood changes}

The distributions of Spearman correlations regarding number of breaks and reported mood score changes are shown on Figure 3. 

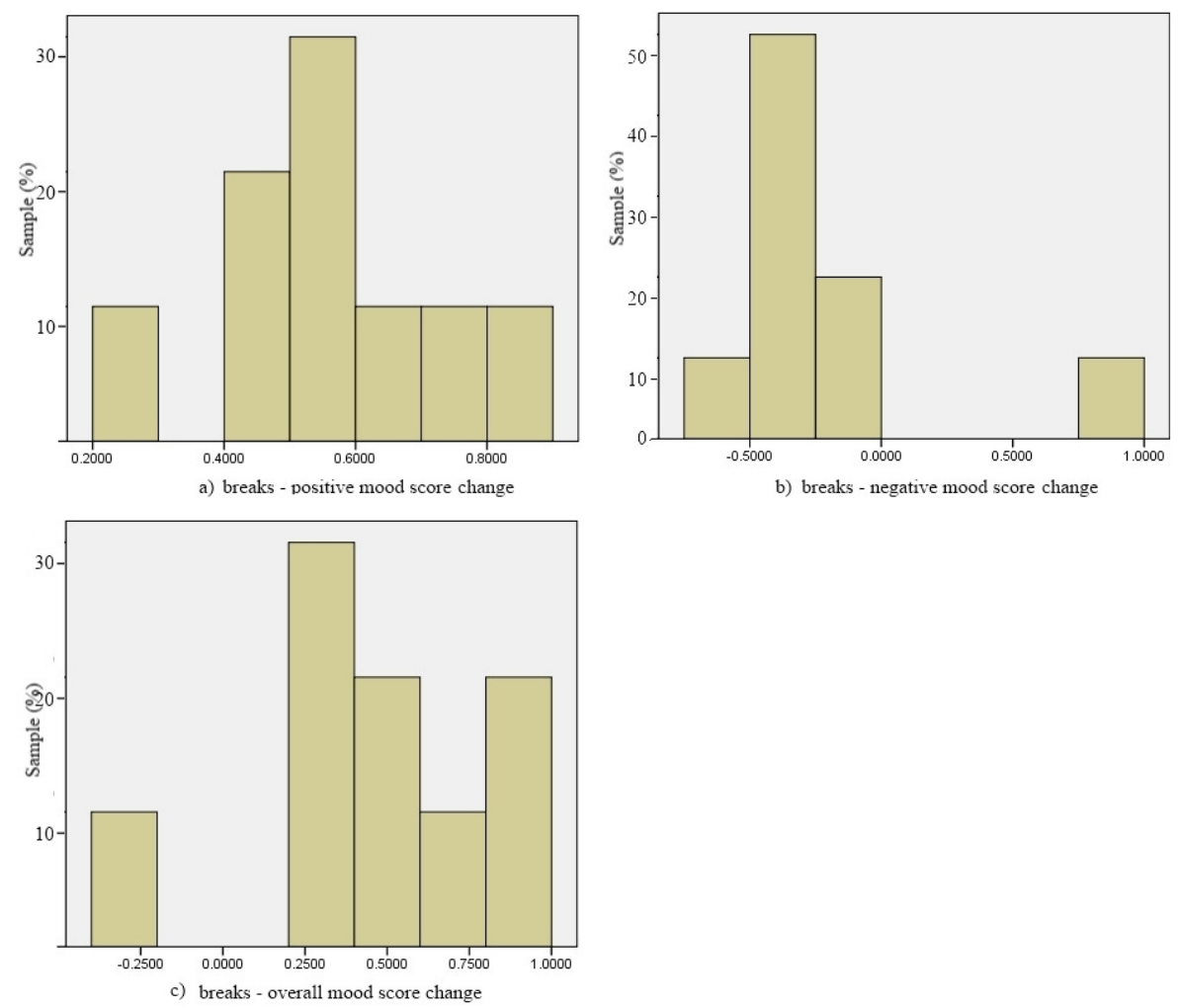

b) breaks - negative mood score change

Figure 3 Distributions of Spearman correlations between number of breaks and a) positive mood, b) negative mood and c) overall mood score change

The mean correlation between number of breaks and positive mood changes was $0.57 \pm 0.15$ ( $\min =0.29$, $\max =0.83$ ) Fig.3a; between number of breaks and negative mood changes was $-0.21 \pm 0.43$ ( $\min =-0.66, \max =0.86$ ) Fig. $3 \mathrm{~b}$; and between number of breaks and overall mood changes was $0.44 \pm 0.35$ ( $\min =-0.27, \max =0.94$ ) Fig.3c. The distribution of Spearman correlations between number of breaks and positive/overall mood changes were significantly greater than $0(t=3.701$ and $t=8.293$ respectively, $\mathrm{P}<0.005, \mathrm{P}<0.001)$. However, the distribution of the Spearman correlation between number of breaks and reported mood change was not significantly different from 0 and it was significantly positively skewed. The results of this study indicate that the subjects were more likely to improve their mood when they had breaks, according to reported positive and overall mood scores. On the other hand, no associations have been found between number of breaks and negative mood score reports. 


\section{Conclusion}

As evidenced from the results presented thus far, there is an evident correlation between breaks and positive and overall mood changes. Non-sedentary work style impacts changes of mood according to positive, negative and overall reported mood scores. Analyzing the psychological implications of prolonged sitting periods will form part of a persuasive feedback application that will be developed to encourage a healthy work style.

Additionally, controlled trials and higher number of participants are necessary to further confirm the findings from this pilot study. While preliminary, this is the first study to link sedentary patterns with mood changes, opening up a research avenue to explore psychological effects of increasing prevalence of sedentary habits.

\section{References}

[1] J.R. Vittengl, and C.S. Holt, "A Time-Series Diary Study of Mood and Social Interaction". Motivation and Emotion, Vol. 22, Number 3 (1998), 255-275.

[2] B. Dardenne, Muriel Dumont, C. Gregoire, M. Sarlet, "Mood and positive testing in social interaction”, European Journal of Social Psychology, vol 41(1), pp 52-63, February 2011.

[3] P. R. Robbins, R. H. and Tanck, "A study of diurnal patterns of depressed mood". Motivation and Emotion, Vol. 11, Number 1 (1987), 37-49.

[4] D. Berry, and J. S. Hansen, "Positive affect, negative affect, and Social Interaction", Journal of Personality and Social Psychology, vol 71(4), October 1996.

[5] L. A. Clark, D. Watson, "Mood and the mundane: Relations between daily life events and selfreported mood", Journal of Personality and Social Psychology. vol. 54(2), pp 296-308,1988.

[6] D. Watson, L. A. Clark, and Curtis W. McIntyre, "Affect, personality, and social activity". Journal of Personality and Social Psychology, 63.6, pp 1011-1025, 1992.

[7] W. F. Stewart, J.A. Ricci, E. Chee, S.R. Hahn, and D. Morganstein, "Cost of lost productive work time along US workers with depression". JAMA, Vol. 289, Number 23 (2003), 3135-3144

[8] J. J. Denissen, L. Butalid, L. Penke, and M. A. Van Aken, "The effects of weather on daily mood: A multilevel approach". Emotion, vol 8, num 5, pp 662-667, 2008.

[9] A. C. Volkers, J. H. M. Tulen, and W.W. Van Den Broek,'Relationships between sleep quality and diurnal variations in mood in healthy subjects".http://www.nswo.nl/userfiles/files/publications/jaarboek-1998/volkers.pdf, (1998), 1-5

[10] M. S. Tremblay, R. C. Colley, T. J. Saunders, G. N. Healy, and Neville Owen,"Psychological and health implications of a sedentary lifestyle". Applied Physiology, Nutrition, and Metabolism, vol. 35(6), pp 725-740, December 2010

[11] J. Kwapisz, G. M. Weiss, S. A. Moore, "Activity recognition using cell phone accelerometers", Human Factors, pp 10-18, 2010. http://storm.cis.fordham.edu/ gweiss/papers/sensorKDD-2010.pdf)

[12] M. J. Mathie, A. C. F. Coster, B. H. Lovell, and B. G. Celler, "Accelerometry: providing an integrated, practical method for long-term, ambulatory monitoring of human movement",Journal of Physiological Measurement, vol 25(2), 2004.

[13] L. A. Clark, D. Watson, J. Leeka, "Diurnal variation in the positive affect", Motivation and Emotion, vol 13(3), pp 205-234, 1999.

[14] A. Adan, and J. Guardia, "Circadian variation of selfreported activations: a multidimensional approach".Chronobiologia, Volume 20, Number 3-4, 233-24, 1993

[15] N. M. Hamburg, C. J. McMackin, A. L.. Huang, S. M. Shenouda. M.E.Widlansky, E. Schulz, et al., "Physical inactivity rapidly induces insulin resistance and microvascular dysfunction in healthy volunteers", Arterioscler. Thromb. Vasc. Biol. 27(12):2650-2656, 2007. 
[16] S. R. Zwart, A. R. Hargens, S. M. Lee, B. R. Macias, D.E. Watenpaugh,K. Tse, S. M. Smith, "Lower body negative pressure treadmill exercise as a countermeasure for bed rest-induced bone loss in female identical twins", Bone, vol 40(2), pp 529-537, 2007.

[17] F .B. Hu, Y. T. Li, G.A. Golditz, W.C. Willett, and J.E. Manson, "Television watching and other sedentary behaviors in relation to risk of obesity and type 2 diabetes mellitus in women", JAMA, vol 289 14), pp 1785-1791, 2003.

[18] G.L. Gierach, S. C.Chang, L. A. Brinton, J.V. Lacey, A. R. Hollenbeck, A.R., A. Schatzkin, M. F. Leitzmann, "Physical activity, sedentary behavior, and endometrial cancer risk in the NIH-AARP Diet and Health Study". Int. J. Cancer, vol 124(9), pp 2139-2147, 2009.

[19] C. K. Martin, T. S., Church, A.M Thompson, C.P. Earnest, S. N. Blair, "Exercise dose and quality of life: a randomized controlled trial”. Arch. Intern. Med. Vol 169(3), pp 269-278, 2009.

[20] A.Sanchez-Villegas, I. Ara, F. Guille'n-Grima, M. Bes-Rastrollo, J.J.Varo-Cenarruzabeitia, M. A. Martı'nez-Gonza'lez, "Physical activity, sedentary index, and mental disorders in the SUN cohort study". Med. Sci. Sports Exerc. Vol 40(5): 827-834, 2008.

[21] A. Matic, A. Popleteev, V. Osmani, O. Mayora, "FM radio for indoor localization with spontaneous recalibration". Journal of Pervasive and Mobile Computing (Elsevier), vol 6(6), pp 642-656, December 2010.

[22] S. L. Curran, M. A.Andykowski, J. L.Studts, "Short form of profile of mood states (POMSSF):Psychometric information”. Psychological Assessment, vol. 7(1),80-83, 1995.

[23] A. Steptoe, and J. Wardle, "Mood and drinking: a naturalistic diary study of alcohol, coffee and tea", Psychopharmacology, vol 141, pp 315-321, 1999.

[24] Aleksandar Matic, Andrei Popleteev, Silvia Gabrielli, Venet Osmani, Oscar Mayora, "Happy or Moody? Why so?: Monitoring daily routines at work and inferring their influence on mood", $5^{\text {th }}$ UbiHealth Workshop in conjuction with UBICOMP2010 Conference, Copenhagen, Denmark, 2010.

[25] Mobile Dev\&Design, http://mobiledecdesign.com [accessed: February 2011]

[26] B. P. Hasler, M. R. Mehl, R. R. Bootzin, and S. Vazire, "Preliminary evidence of diurnal rhythms in everyday behaviors associated with positive affect". Journal of Research in Personality, vol. 42 (6), pp 1537-1546, 2008.

[27] Z. Zeng, M. Pantic, G. I. Roisman, and T. S. Huang, "A Survey of Affect Recognition Methods: Audio, Visual and Spontaneous Expressions". IEEE Transactions on Pattern Analysis and Machine Intelligence, vol. 31(1) 39-58, 2009

[28] R.E.Thayer, "Problem perception, optimism, and related states as a function of time of day (diurnal rhythm) and moderate exercise: Two arousal systems in interaction". Motivation and Emotion, vol. 11(1), pp 19-36, 1987.

[29] Wood, C., and Magnello, M.E. Diurnal changes in perceptions of energy and mood. Journal of the Royal Society of Medicine, vol. 85(4), pp 191-194, 1992.

[30] A.A. Stone, J. M Smyth, T.Pickering, and J. Schwartz, "J. Daily mood variability: Form of diurnal patterns and determinants of diurnal patterns. Journal of Applied Social Psychology, Vol. 26, Issue 14 pp 1286-1305, 2006

[31] E.H. Hon, S.T. Lee, "Elecetronic Evaluation of the fetal heart rate patterns preceding fetal death, further observations". Am J Obstet Gynec col. 87, pp 814-826, 1965.

[32] Biocom Technologies - Heart Rate Variability basics. http://www.biocomtech.com/hrv-science/heartrate-variability-basics [acessed: February 2011]

[33] W. Wu, J. Lee, H. Chen, "Estimation of heart rate variability changes during different visual stimulations using non-invasive real-time ECG monitoring system", International Joint Conference on Bioinformatics, System Biology and Intelligent Computing, 2009.

[34] M. Malik, for the Task Force of the ESC and NASPE, "Heart rate variability: standards of measurement, physiological interpretation and clinical use", Circulation, vol. 93, no. 5, pp. 1043$1065,1996$.

[35] M. Matsuanaga, T. Isowa, M. Miyakoshi, N. Kanayama, H. Mukarami, S. Fukuyama et. Al, "Associations among positive mood, brain, and cardiovascular activities in an affectively positive situation”. Brain Research, vol. 1263(), pp 93-103, 2009.

[36] F.C.M. Geisler, N. Vennewald, T. Kubiak and H. Weber, "The impact of heart rate variability on subjective well-being is mediated by emotion regulation". Personality and Individual Differences, vol. 49(7), pp 723-728, 2010. 
[37] J. Taelman, S. Vandeput, A. Spaepen, and S. Van Huffel, "Influence of Mental Stress on Heart Rate and Heart Rate Variability”. ECIFMBE 2008, IFMBE Proceedings 22, pp. 1366-1369, 2008.

[38] Shimmer - Wireless Sensing Solutions, http://www.shimmer-research.com [accessed: February 2011]

[39] D. O. Olguin, P. A. Gloor, and A. S. Pentlandm "Capturing Individual and Group Behavior with Wearable Sensors", AAAI Spring Symposium on Human Behavior Modeling, 2009.

[40] Fishbach, K., Schoder, D., and Gloor, P.A. Analysis of Informal Communications Networks - A case study. Business \& Information Systems Engineering, Volume 1, Number 2 (2009), 140-149

[41] RFID Journal, http://www.rfidjournal.com/article/view/4542/1[accesed May 2011]

[42] Tudor-Locke CE, Myers AM. Challenges and opportunities for measuring physical activity in sedentary adults.Sports Med 2001;31:91-100

[43] G. Jennings, L. Nelson, P. Nestel, M. Esler, P. Korner, D. Burton, and J. Bazelmans,'The effects of changes in physical activity on major cardiovascular risk factors, hemodynamics, symphatetic function, and glucose utilization in man: a controlled study of four levels of activity", Circulation, Vol 73, pp 30-40, 1986.

[44] L. Reagan, "Insulin signaling effects on memory and mood", Current Opinion in Parmacology, Col 7, pp 633-637, 2007.

[45] A. Wells, N. W. Read, and A. Macdonald, "Effects of Carbohydrate and Lipid on Resting Energy Expenditure, Heart Rate, Sleepiness, and Mood", Physiological Behavior, Vol 63(4), pp 621-628, 1998.

[46] L. A. Gonder-Fredericl, D. J. Cox, S. A. Bobbitt, and J. Pennebaker, "Mood changes associated with blood glucose fluctuations in insulin-dependend diabetes mellitus", Health Psychology, Vol. 8(1), pp 45-59, 1989.

[47] Hamilton, M.T., Hamilton, D.G., and Zderic, T.W. 2007. Role of low energy expenditure and sitting in obesity, metabolic syndrome, type 2 diabetes, and cardiovascular disease. Diabetes,56(11): 26552667. doi:10.2337/db07-0882. PMID:17827399

[48] S. G. Trost, K. L. Mciver, and R. R. Pate, "Conducting accelerometer-based activity assessments in field-based research", Medicine\&Science in Sports\&Exercise, DOI: 10.1249/01.mss0000185657.86065.98, 2005

[49] J. Sivula, W. B. Lake, R. A. Betzold, "Method and apparatus for implementing activity sensing in a pulse generator", US Patent number 5.052.388, 1989

[50] J. M. Smyth and A. A. Stone, "Ecological momentary assessment research in behavioral medicine". Journal of Happiness Studies, Vol. 4, pp 35-52, 2003

[51] G. N. Healy, D. W. Dunstan, J. Salmon, and E. Cerin, "Breaks in sedentary time: Beneficial associations with metabolic risk". Diabetes care, Vol. 31, number 4, 2008 